\title{
Critical $k$-very ampleness for abelian surfaces
}

\author{
Wafa Alagal and Antony Maciocia
}

\begin{abstract}
Let $(S, L)$ be a polarized abelian surface of Picard rank 1, and let $\phi$ be the function which takes each ample line bundle $L^{\prime}$ to the least integer $k$ such that $L^{\prime}$ is $k$-very ample but not $(k+1)$-very ample. We use Bridgeland's stability conditions and FourierMukai techniques to give a closed formula for $\phi\left(L^{n}\right)$ as a function of $n$, showing that it is linear in $n$ for $n>1$. As a by-product, we calculate the walls in the Bridgeland stability space for certain Chern characters.
\end{abstract}

\section{Introduction}

The notion of $k$-very ampleness was introduced in the 1980s initially to understand the idea of higher-order embeddings. Weaker notions of $k$-spanned (see [BS1], [BFS]) and $k$-jet ampleness (see [BS2]) were also considered. The definitions given all relate to asking that, for a variety $V$ and ample line bundle $L$ on $V$, the natural map $\Gamma(L) \rightarrow \Gamma(L / \mathcal{I})$ surjects for certain classes of sheaf $\mathcal{I}$ of 0 -subschemes of $V$. The notions differ in how fat points are treated. In this paper we only consider the strongest notion of $k$-very ampleness. Since $k$-very ampleness implies $(k-1)$-very ampleness, it is natural to consider the critical value of $k$ when a line bundle $L$ is $k$-very ample but not $(k+1)$-very ample. We shall denote this by $\phi(L)$. In the case when the Néron-Severi group is generated by a single element (as we shall be assuming), we can view the function $\phi$ as a function of a positive integer. It is then natural to hope that $\phi\left(L^{n+1}\right)$ is related to $\phi\left(L^{n}\right)$. Unfortunately, $k$-very ampleness is not very well behaved with respect to tensoring. It is not even clear that $\phi\left(L^{n}\right)$ can be expressed as a nice function of $n$. It is typically bounded above by a degree $\operatorname{dim} V$ polynomial and below by a linear function of $n$, and one would expect it to be eventually polynomial for large enough $n$. We show, in fact, that $\phi\left(L^{n}\right)$ equals $c_{1}(L)^{2}(n-1)-2$ for $n \geq 2$ for a polarized abelian surface $(S, L)$ for which $\mathrm{NS}(S)=\langle L\rangle$.

One type of variety where much progress has been made is abelian varieties. There are a number of, by now, classical results on the very ampleness of line bundles. For example, $L^{3}$ is always very ample for any ample line bundle $L$. A little more recently, Debarre, Hulek, and Spandaw showed that a suitably

Kyoto Journal of Mathematics, Vol. 56, No. 1 (2016), 33-47

DOI $10.1215 / 21562261-3445147$, C 2016 by Kyoto University

Received March 11, 2014. Revised May 22, 2014. Accepted December 3, 2014.

2010 Mathematics Subject Classification: Primary 14C20; Secondary 14D22, 14K99. 
generic $(1, d)$ polarization on a $g$-dimensional abelian variety is very ample for $d>2^{g}$. For the case $g=2$ and Picard rank 1, this was extended by Bauer and Szemberg [BS] to compute $\phi(1)$ (see Proposition 4.1 below for the details). The issue of $k$-very ampleness and $k$-spannedness was also studied by Terakawa [T2, Corollary 4.2], who showed that they coincide for a polarized abelian surface. He also gave necessary and sufficient conditions for when a line bundle is $k$-very ample (see [T1], [T2, Theorem 1.1]), but these depend on the existence of certain divisors and the resulting inequalities are tricky to solve.

There is a clear relation between $k$-very ampleness and so-called weak index theorem conditions arising in Fourier-Mukai theory for abelian varieties. These ideas have been extended by Pareschi and Popa [PP1], who introduced the notion of $M$-regularity and related it to $k$-jet ampleness in [PP2].

This paper is organized as follows. For the rest of the Introduction we define the $\phi$-function and recall some facts already established in the literature. We also recall some facts about Fourier-Mukai transforms and deduce some easy results about $\phi$. In Section 2 we give a brief introduction to Bridgeland's stability conditions needed to prove the main theorem in this paper. In Section 3 we recall the notion of walls and show that for the Chern character $(r, l, \chi)$ there are no walls. We also use general stability machinery to provide a useful technical lemma (Lemma 3.5) needed to prove our main theorem. In the final section, we show how to use the technical lemma to bound $\phi$ from above and then prove that the bound is sharp by computing walls in the stability space associated to the Chern character $\left(1, n l,(n-1)^{2} d+d+1\right)$. We then induct on $n$ to deduce the main theorem, making use of our technical lemma again.

THEOREM 4.3

If $(S, L)$ is a polarized abelian surface with $\mathrm{NS}(S)=\langle L\rangle$ and $c_{1}(L)^{2}=2 d$, then $\phi\left(L^{n}\right)=2(n-1) d-2$.

\section{1. $k$-very ample}

Let $V$ be a complete algebraic variety of dimension $g$ over an algebraic closed field $\mathbb{K}$, let $X$ be a 0 -dimensional subscheme of $V$ with $|X|=d=\operatorname{dim}\left(H^{0}\left(\mathcal{O}_{X}\right)\right)$, and let $L$ be an invertible sheaf on $V$.

\section{DEFINITION 1.1}

For each 0 -scheme $X$ on $V$ we can consider the restriction map $\rho_{X}$ to $X$ for the space of sections of $L$, which fits into the exact sequence:

$$
\begin{aligned}
0 & \rightarrow H^{0}\left(V, L \otimes \mathcal{I}_{X}\right) \rightarrow H^{0}(V, L) \stackrel{\rho_{X}}{\rightarrow} H^{0}\left(\mathcal{O}_{X}\right) \rightarrow H^{1}\left(V, L \otimes \mathcal{I}_{X}\right) \\
& \rightarrow H^{1}(V, L) \rightarrow 0 .
\end{aligned}
$$

Then $L$ is called $k$-very ample if $\rho_{X}$ is surjective for all purely 0 -dimensional subschemes $X$ of length $|X| \leq k+1$. 


\section{REMARK 1.2}

The following statements follow easily from the definition:

- $L$ is 0 -very ample if and only if $L$ is generated by a global section.

- $L$ is 1 -very ample if and only if it is very ample.

- If $L$ is $k$-very ample, then $L$ is $(k-1)$-very ample.

Let $\operatorname{Amp}(S)$ be the ample cone of $S$. By the properties above there exists an integer $k$ for all $L \in \operatorname{Amp}(S)$ such that $L$ is $k$-very ample but not $(k+1)$-very ample.

\section{DEFINITION 1.3}

Define a map

$$
\phi: \operatorname{Amp}(S) \rightarrow \mathbb{Z}_{\geq-1},
$$

which takes $L$ into the least integer $k$ such that $L$ is $k$-very ample but not $(k+1)$ very ample. Define $\phi_{L}(n):=\phi\left(L^{n}\right)$, which we write as $\phi(n)$ if $L$ is understood.

There is no obvious reason why this should be a good function of $n$ for any variety, and even for $\mathbb{P}^{2}$, it is hard to compute. Specific values for some varieties are, however, well known.

\section{EXAMPLE 1.4}

Let $(V, L)$ be a principally polarized abelian variety. Then $\phi_{L}(2)=0$.

The following lemma, indirectly proved in [BS, Propositions 3.2, 3.3], gives the value of $\phi_{L}(1)$, and we will re-prove it (in slightly greater generality for arbitrary type $\left.\left(d_{1}, d_{2}\right)\right)$ in Section 4 in the spirit of this paper.

PROPOSITION 1.5 ([BS])

If $L$ is an ample line bundle on an abelian surface $X$ with $\operatorname{NS}(X)=\langle L\rangle$ and $c_{1}(L)^{2}=2 d$, then

$$
\phi_{L}(1)=\left\lfloor\frac{d-3}{2}\right\rfloor
$$

Upper and lower bounds for $\phi$ are also known. It is clear that if $H^{1}(L)=0$ (as is the case, for example, for an ample line bundle on an abelian variety), then an upper bound for $\phi(n)$ can be given by $\chi\left(L^{n}\right)-1$ since $\chi\left(L^{n} \otimes \mathcal{I}_{X}\right)=\chi\left(L^{n}\right)-|X|$. For a polarized abelian surface, this is $n^{2} d-1$. A more careful analysis (from the condition $L^{2} \geq 4 k+6$ in [T2, Theorem 1.1]) gives $\frac{1}{2}\left(n^{2} d-3\right)$, which is consistent with Theorem 4.3. A nontrivial lower bound is much harder to come by but Reider's theorem (for the most useful version, see [AB, Section 2]) provides one, at least when certain divisors do not exist, as it says that if $c_{1}\left(L^{n}\right)^{2}>(k+2)^{2}$, then $L^{n}$ is $k$-very ample. If we apply this to the Picard rank 1 abelian surface 
case where such divisors do not exist, we see that $\phi(n) \geq\lceil\sqrt{2 d} n\rceil-3$. But this is not even sharp for $d=1$ and $n=2$.

Reider's theorem arose in the situation where $L \otimes \mathcal{I}_{Z}$ is used to construct vector bundles of rank 2. Key in his construction is the Bogomolov inequality for semistable sheaves. We will also use this in various places and recall it here (see [HL, Theorem 7.3.1] for a proof).

\section{DEFINITION 1.6}

A torsion-free sheaf $E$ is $\mu$-stable (resp., $\mu$-semistable) with respect to $l$ if for each proper subsheaf $F$ we have

$$
\mu(F)<\mu(E) \quad(\mu(F) \leq \mu(E)),
$$

where $\mu(E)=c_{1}(E) \cdot l^{g-1} / r(E)$.

Recall the Bogomolov inequality, which provides us with a very useful necessary condition for $\mu$-semistability.

\section{PROPOSITION 1.7}

Let $V$ be a smooth projective variety of dimension $n$, and let $l$ be an ample divisor on $V$. If $E$ is a $\mu$-semistable (with respect to $l$ ) torsion sheaf of rank $r$ on $V$, then

$$
(r-1) c_{1}^{2}(E) \cdot l^{n-2} \leq 2 r c_{2}^{2}(E) \cdot l^{n-2} .
$$

For the case of an abelian surface this reads as

$$
2 r(E) \chi(E) \leq c_{1}^{2}(E) .
$$

We will also need to consider a finer stability for sheaves.

\section{DEFINITION 1.8}

A torsion-free sheaf $E$ is Gieseker stable (resp., Gieseker semistable) with respect to $l$ if for each subsheaf $F$ we have

$$
P(F)<P(E) \quad(P(F) \leq P(E)),
$$

where $P(E)=\chi\left(E \otimes L^{n}\right) / r(E)$ is the reduced Hilbert polynomial.

We let $\mathcal{M}_{\text {ch }}^{\text {GS }}$ denote the moduli space of Gieseker semistable sheaves on $S$ with Chern character ch (or, more generally, Simpson semistable sheaves when the rank is 0$)$. The virtual dimension of $\mathcal{M}_{(r, c, \chi)}^{\mathrm{GS}}$ is $2 c^{2} d-2 r \chi+2$ and these spaces are nonempty exactly when this dimension is at least 2 (i.e., exactly when the Bogomolov inequality holds). The case when the dimension is exactly 2 was proved by Mukai [Muk, Proposition 6.22], and the remaining cases are dealt with in [Y, Theorem 0.1]. We will need nonemptiness specifically for the cases where $c= \pm l$ and $\chi=1$ or $\chi=2$, which are studied in detail in [Y, Section 6]. 


\subsection{Fourier-Mukai transforms}

Let $V$ and $\hat{V}$ be smooth projective varieties. Consider the flat projections $V \stackrel{\pi}{\leftarrow}$ $V \times \hat{V} \stackrel{\hat{\pi}}{\rightarrow} \hat{V}$. Let $\mathcal{P} \in D(V \times \hat{V})$, where $D(V \times \hat{V})$ denotes the derived category of bounded complexes of coherent sheaves on $V \times \hat{V}$. The Fourier-Mukai transform $\Phi$ is the functor

$$
\Phi: D(V) \rightarrow D(\hat{V})
$$

which takes $A$ into $R \pi_{*}\left(L \hat{\pi}^{*} A \stackrel{L}{\otimes} \mathcal{P}\right)$ (see [Huyl]). Denote its cohomology by $\Phi^{i}$. In fact, we shall only consider the classical Fourier-Mukai transform where $\mathcal{P}$ is the Poincaré bundle on an abelian surface $V=S$. Then $\Phi$ has a quasi-inverse given (up to shift) by the transform

$$
\hat{\Phi}: D(\hat{S}) \rightarrow D(S)
$$

with kernel $\hat{\mathcal{P}} \in D(\hat{S} \times S)$, where $\hat{\mathcal{P}}=s^{*} \mathcal{P}$ and $s: S \times \hat{S} \rightarrow \hat{S} \times S$ is $\left(\begin{array}{cc}0 & -1 \\ 1 & 0\end{array}\right)$.

DEFINITION 1.9

An object $E$ satisfies $\mathrm{WIT}_{n}$ if $\Phi^{i}(E)=0$ for all $i \neq n$.

\section{DEFINITION 1.10}

An object $E$ satisfies $\operatorname{IT}_{n}$ if $H^{i}\left(E \otimes \mathcal{P}_{\hat{x}}\right)=0$ for all $\hat{x} \in \hat{S}$, the dual of $S$, and $i \neq n$. In this case, $\Phi^{n}(E)$ is a locally free sheaf.

\section{EXAMPLE 1.11 ([Mum])}

Any ample line bundle $L$ on an abelian variety is $\mathrm{IT}_{0}$. Any sheaf which is $\mathrm{WIT}_{0}$ is automatically $\mathrm{IT}_{0}$ by the semicontinuity theorem.

\section{PROPOSITION 1.12}

If $(S, \Phi)$ is an abelian variety and $L$ is $\mathrm{IT}_{0}$, then $L$ is $k$-very ample if and only if $L \otimes \mathcal{I}_{X}$ is $\mathrm{WIT}_{0}$ (and hence $\mathrm{IT}_{0}$ ) for all 0-dimensional subschemes $X$ of length $|X| \leq k+1$.

Proof

To prove the "if" part of the proposition, suppose that $L$ is $k$-very ample, and suppose that $L \otimes \mathcal{I}_{X}$ is not $\mathrm{WIT}_{0}$ for some purely 0 -dimensional subscheme $X$ of length $|X| \leq k+1$, so there exists $\hat{x} \in \hat{S}$ such that $H^{1}\left(L \mathcal{P}_{\hat{x}} \mathcal{I}_{X}\right) \neq 0$. Pick $x$ for which $\hat{x}=\psi_{L}(x)$, where $\psi_{L}: S \rightarrow \hat{S}$ is the étale map which takes $x$ into $\tau_{x}^{*} L \otimes L^{-1}$; then $H^{1}\left(\tau_{-x}^{*}(L) \mathcal{I}_{X}\right) \neq 0$ and so $H^{1}\left(\tau_{-x}^{*}\left(L \otimes \mathcal{I}_{\tau_{-x} X}\right)\right) \neq 0$. Hence, $H^{1}\left(L \otimes \mathcal{I}_{\tau_{-x} X}\right) \neq 0$, where $\left|\tau_{-x} X\right| \leq k+1$, and this contradicts the assumption.

To prove the "only if" part of the proposition, since $L \otimes \mathcal{I}_{X}$ is $\mathrm{WIT}_{0}$ for all purely 0-dimensional subschemes $X$ of length $|X| \leq k+1$, we have $H^{1}\left(L \otimes \mathcal{I}_{X}\right)=$ 0 . Hence, $L$ is $k$-very ample by definition. 
PROPOSITION 1.13

Let $(S, L)$ be an irreducible principally polarized abelian surface. Then $L^{n}$ is not $(2 n-3)$-very ample.

Proof

Let $X$ be a 0 -dimensional subscheme of $D_{L}$ of length $2(n-1)$. Then we have a sequence

$$
0 \rightarrow L^{n-1} \rightarrow L^{n} \otimes \mathcal{I}_{X} \rightarrow Q \rightarrow 0 .
$$

Suppose that $Q$ is $\mathrm{IT}_{0}$. The Chern character of $Q$ is $\operatorname{ch}(Q)=(0, l,(2 n-1)-|X|)$. The transform of $Q$, denoted $\hat{Q}$, has the Chern character $\operatorname{ch}(\hat{Q})=((2 n-1)-$ $|X|,-l, 0)=(1,-l, 0)$, but $\hat{Q}$ is locally free, which is impossible. So $Q$ is not $\mathrm{IT}_{0}$ and then $L^{n} \otimes \mathcal{I}_{X}$ is not $\mathrm{IT}_{0}$.

Such $X$ we call collinear, as $H^{0}\left(L \otimes \mathcal{I}_{X}\right) \neq 0$, so there exists $x \in S$ such that $X \subset \tau_{x} D_{L}$, a translation of the polarization divisor.

\section{COROLLARY 1.14}

Let $(S, L)$ be an irreducible principally polarized abelian surface. Then $\phi(n) \leq$ $2 n-4$ for $n \geq 2$.

\section{Bridgeland stability conditions}

Now we will give a brief review of Bridgeland's stability conditions (see [Bri]). We follow the conventions of [Mac] and $[\mathrm{AB}]$. Define for any $s \in \mathbb{R}$ the following:

$$
\begin{aligned}
& F_{s}=\left\{E \in \operatorname{Coh}_{S} \mid E \text { is torsion-free and } \mu_{+}(E) \leq 2 d s\right\}, \\
& T_{s}=\left\{E \in \operatorname{Coh}_{S} \mid E \text { is torsion or } \mu_{-}(E / \operatorname{tors}(E))>2 d s\right\},
\end{aligned}
$$

where $\mu_{+}(E)$ is the slope of the largest slope $\mu$-destabilizing subsheaf of $E$ and $\mu_{-}(E)$ is the slope of the lowest slope $\mu$-destabilizing quotient of $E$. We set

$$
\mathcal{A}_{s}=\left\{A \in D(S) \mid A^{i}=0, i \notin\{0,-1\}, H^{-1}(A) \in F_{s}, H^{0}(A) \in T_{s}\right\} .
$$

A group homomorphism $Z_{s, t}$ takes the Chern character $\operatorname{ch}(A)$ into

$$
\begin{aligned}
Z_{s, t}(A) & =\left\langle e^{(s+t i) l}, \operatorname{ch}(A)\right\rangle \\
& =-\chi+2 d c s+d r\left(t^{2}-s^{2}\right)+2 t d i(c-r s) .
\end{aligned}
$$

For each $A \in \mathcal{A}_{s}$ the slope $\mu_{s, t}(A) \in \mathbb{Q} \cup\{+\infty\}$ of $A$ is given by

$$
\begin{aligned}
\mu_{s, t}(A) & =-\frac{\operatorname{Re}\left(Z_{s, t}(A)\right)}{\operatorname{Im}\left(Z_{s, t}(A)\right)} \\
& =\frac{\chi-2 d c s-d r\left(t^{2}-s^{2}\right)}{2 t d(c-r s)}
\end{aligned}
$$

if $\operatorname{Im}\left(Z_{s, t}(A)\right) \neq 0$ and $+\infty$ otherwise. 


\section{DEFINITION 2.1}

We say that $E \in \mathcal{A}_{s}$ is $\sigma_{s, t^{-}}$stable (resp., $\sigma_{s, t^{-}}$semistable) if for all injections $F \rightarrow E$ in $\mathcal{A}_{s}$ we have $\mu_{s, t}(F)<\mu_{s, t}(E)$ (resp., $\mu_{s, t}(F) \leq \mu_{s, t}(E)$ ). It is well known that these give sensible stability conditions on any smooth surface. When $s=0$, we will write $\sigma_{t}$ for $\sigma_{0, t}$.

Then the slope of $E=L^{n} \otimes \mathcal{I}_{X}$ where $\operatorname{ch}(E)=\left(1, n l, n^{2} d-|X|\right)$ is

$$
\mu_{s, t}(E)=\frac{n^{2} d-|X|-2 d n s-d\left(t^{2}-s^{2}\right)}{2 t d(n-s)} .
$$

Note that $n>s$ as $E \in T_{s}$.

\section{REMARK 2.2}

Now suppose that $F \in \mathcal{A}_{s}$ with $\operatorname{ch}(F)=(r, c l, \chi)$ destabilizes $L^{n} \otimes \mathcal{I}_{X}$. Then we have a short exact sequence $F \rightarrow E \rightarrow Q$ in $\mathcal{A}_{s}$. Taking cohomology we see that $H^{-1}(F)=0$. Then $F \in T_{s}$ and so $c>r s$. Notice also that $H^{-1}(Q) \in F_{s}$ is torsion-free, and since $0 \rightarrow H^{-1}(Q) \rightarrow F \rightarrow E$ is exact, $F$ is also torsion-free.

We also have

$$
\mu_{s, t}(F)-\mu_{s, t}(E)>0
$$

Therefore

$$
\frac{\chi-2 d c s+d r\left(s^{2}-t^{2}\right)}{2 t d(c-r s)}-\frac{n^{2} d-|X|-2 d n s-d\left(t^{2}-s^{2}\right)}{2 t d(n-s)}>0 .
$$

Define $f(F, E)$ to be the numerator of (2.3). Then

$$
\begin{aligned}
f(F, E)= & \left(\chi-2 d c s+d r\left(s^{2}-t^{2}\right)\right)(n-s) \\
& -\left(n^{2} d-|X|-2 d n s-d\left(t^{2}-s^{2}\right)\right)(c-r s) \\
= & (n-s) \chi-c\left(n^{2} d-|X|-d\left(s^{2}+t^{2}\right)\right)+r\left(n^{2} d s-|X| s-d n\left(s^{2}+t^{2}\right)\right) .
\end{aligned}
$$

We shall be most interested in the case when $s=0$. Then the destabilizing condition becomes

$$
f(F, E)=n \chi-c\left(n^{2} d-|X|-d t^{2}\right)-d n r t^{2}>0 .
$$

Therefore

$$
n \chi-c n^{2} d+c|X|>(n r-c) d t^{2},
$$

and $c \leq n r$ because $\mu\left(H^{-1}(Q)\right) \leq 0$ and $\mu(F) \leq \mu\left(F / H^{-1}(Q)\right) \leq \mu(E)$. Hence, a necessary condition for the existence of such a destabilizing object is

$$
n \chi-c n^{2} d+c|X|>0
$$

Recall from [Bri, Proposition 14.2] that, in the large volume limit as $t \rightarrow \infty$, the $\sigma_{t}$-semistable objects $E$ with $\mu(E)>0$ are exactly the Gieseker semistable sheaves (when $s=0$ ). The case when $\mu(E)<0$ is similar. In this case, $r(E)<0$ when $s=0$. 
PROPOSITION 2.3

Suppose that $F \in \mathcal{A}_{0}$ with $\mu(F)<0$. Then $F$ is $\sigma_{t}$-semistable for all $t \gg 0$ if and only if $H^{0}(F)$ is supported in dimension 0 and $H^{-1}(F)$ is a Gieseker semistable vector bundle.

Proof

The proof follows in same way as that of [Bri, Proposition 14.2] by observing that if $E$ is Bridgeland stable for all $t \gg 0$, then $H^{0}(E)$ must be supported in dimension 0; otherwise, $\mu_{0, t}\left(H^{0}(E)\right)$ is finite and $H^{0}(E)$ destabilizes $E$ for $t \gg 0$. Moreover, $H^{-1}(E)[1]$ is locally free since

$$
0 \rightarrow \mathcal{O}_{Z} \rightarrow H^{-1}(E)[1] \rightarrow H^{-1}(E)^{* *}[1] \rightarrow 0
$$

is a short exact sequence in $\mathcal{A}_{0}$ and then $\mathcal{O}_{Z} \rightarrow E$ would destabilize $E$. The fact that $H^{-1}(E)$ is Gieseker semistable follows in the same way as in [Bri].

\section{REMARK 2.4}

An alternative approach can be seen using an observation of Yanagida and Yoshioka [YY, Proposition 2.6], who show that the Bridgeland stability is preserved under [1] $\Delta$, where $\Delta(E)=\mathbf{R} \mathcal{H o m}\left(E, \mathcal{O}_{S}\right)$ at least when $c_{1} \cdot \ell \neq 0$. So if $F$ is $\sigma_{t}$-semistable, then $F^{\vee}$ is $\sigma_{t}$-semistable and $\mu\left(F^{\vee}\right)>0$. Then [Bri, Proposition 14.2] implies that $F^{\vee}$ is a Gieseker semistable sheaf. Therefore, $F^{\vee \vee} \cong F$ takes the required form. In particular, observe that $H^{0}(F) \neq 0$ exactly when $F^{\vee}$ is not locally free.

\section{REMARK 2.5}

Huybrechts [Huy2] showed that $\Phi[1]$ preserves $\mathcal{A}_{0}$ and it can also be shown (see, e.g., [MM, Proposition 3.2]) that $E \in \mathcal{A}_{0}$ is $\sigma_{t^{-}}$stable if and only if $\Phi(E)$ is $\sigma_{1 / t^{-}}$ stable. (Semistability is similar.)

\section{Walls and moduli spaces}

\section{DEFINITION 3.1}

We let $\mathcal{M}_{\mathrm{ch}}^{\mathrm{BS}, t}$ denote the moduli space of $\sigma_{t}$-semistable objects in $\mathcal{A}_{0}$.

It is well known that these spaces are projective varieties for a wide selection of spaces. For the case of K3 and abelian surfaces it follows from the sliding down the wall trick of [Mac] and applying Remark 2.5, but it was also independently observed by Minamide, Yanagida, and Yoshioka [MYY, Theorem 5.3] in the Picard rank 1 case and proved more generally for K3 surfaces in [BM, Theorem 1.3].

For example, in the large volume limit as $t \rightarrow \infty, \mathcal{M}_{\mathrm{ch}}^{\mathrm{BS}, t}=\mathcal{M}_{\mathrm{ch}}^{\mathrm{GS}}$ when $c_{1}(\mathrm{ch})$. $l>0$ and $r(\mathrm{ch})>0$. Equality here means that the points represent exactly the same objects in $\operatorname{Coh}(S) \cap \mathcal{A}_{0}$ up to isomorphism. When the slope is negative, Remark 2.4 implies that the large volume limit of $\mathcal{M}_{\mathrm{ch}}^{\mathrm{BS}, t}$ for large $t$ is given by objects $E^{\vee}[1]$, where $[E] \in \mathcal{M}_{\mathrm{ch}^{*}:=\left(\mathrm{ch}_{0},-\mathrm{ch}_{1}, \mathrm{ch}_{2}\right)}^{\mathrm{GS}}$ and so $\mathcal{M}_{\mathrm{ch}}^{\mathrm{BS}, t} \cong \mathcal{M}_{\mathrm{ch}^{*}}^{\mathrm{GS}}$. 
It may happen for some value of $t$ that the two moduli spaces are not equal. In fact, there will be a strictly decreasing sequence $t_{0}, t_{1}, \ldots$ of values of $t$ on either side of which $\mathcal{M}_{\mathrm{ch}}^{\mathrm{BS}, t}$ differ. We call these walls. (Sometimes they are called miniwalls when we fix s.) Our aim will be to identify these walls when $\mathrm{ch}=(0, l, \chi)$ and $\mathrm{ch}=\left(1, n l, n^{2} d-|X|\right)$. In the first case we show that there are no walls which generalize [Mac, Proposition 4.2].

LEMMA 3.2

For $s=0$ there are no walls for $\mathrm{ch}=(r, l, \chi)$ for any $\chi, r \in \mathbb{Z}$.

Proof

If $E \in \mathcal{A}_{0}$, then $c_{1}(E) \cdot l \geq 0$ and $c_{1}(E)=0$ if and only if $\mu_{0, t}(E)=\infty$. If $E$ has $c_{1}(E)=l$ and sits in a short exact sequence

$$
0 \rightarrow K \rightarrow E \rightarrow Q \rightarrow 0
$$

in $\mathcal{A}_{0}$, then either

(i) $c_{1}(K)=0$, in which case the sequence destabilizes $E$ for all $t$; or

(ii) $c_{1}(Q)=0$, in which case the sequence does not destabilize for any $t$.

It follows that there are no walls in $\mathcal{A}_{0}$.

REMARK 3.3

It follows that $\mathcal{M}_{(0, l, \chi)}^{\mathrm{GS}}=\mathcal{M}_{(0, l, \chi)}^{\mathrm{BS}, t}$ for all $t$ and $s=0$ and, by Remark 2.5, ch $=$ $(\chi,-l, 0)$ also has no walls for $s=0$. Hence, for all $t>0$,

$$
\mathcal{M}_{(\chi, l, 0)}^{\mathrm{BS}, t}=\mathcal{M}_{(\chi, l, 0)}^{\mathrm{GS}}, \quad \text { when } \chi \geq 0,
$$

and

$$
\mathcal{M}_{(\chi, l, 0)}^{\mathrm{BS}, t}=\Delta \mathcal{M}_{(-\chi, l, 0)}^{\mathrm{GS}}[1], \quad \text { when } \chi<0 .
$$

DEFINITION 3.4

We say that the moduli space $\mathcal{M}_{(r, c l, \chi)}^{\mathrm{BS}, t}$ of Bridgeland stable sheaves of Chern character $(r, c l, \chi)$ satisfies $\mathrm{IT}_{0}$ (resp., $\mathrm{WIT}_{0}$ ) if and only if, for each $E$ representing an object of $\mathcal{M}_{(r, c l, \chi)}^{\mathrm{BS}}, E$ satisfies $\mathrm{IT}_{0}$ (resp., $\mathrm{WIT}_{0}$ ).

For example, $\mathcal{M}_{\left(1, n l, n^{2} d-k\right)}^{\mathrm{BS}, t}$ is $\mathrm{IT}_{0}$ for all $t$ if and only if $L^{n}$ is $(k-1)$-very ample and so $\phi_{L}(n) \geq k-1$. Note that if $\mathcal{M}$ is a fine moduli space and $[E] \in \mathcal{M}$, then $E$ is $\mathrm{IT}_{0}$ if and only if all $F \in[E] \in \mathcal{M}$ are $\mathrm{IT}_{0}$. This may not be true when the moduli space is not fine (and there exist non-Gieseker stable sheaves) because the $\mathrm{IT}_{0}$ condition is not preserved by $S$-equivalence. However, the moduli spaces we consider below will all be fine.

The following technical result will be useful in the next section.

LEMMA 3.5

We have that $\mathcal{M}_{(0, l, \chi)}^{\mathrm{GS}}$ is $\mathrm{IT}_{0}$ if and only if $\chi \geq d+1$. 
Proof

We use Proposition 2.3, Remarks 2.4 and 3.3, and Lemma 3.2 to give isomorphisms

$$
\mathcal{M}_{(0, l, \chi)}^{\mathrm{GS}} \stackrel{\Phi[1]}{\cong} \mathcal{M}_{-(\chi,-l, 0)}^{\mathrm{BS}, t} \stackrel{[1] \Delta}{\cong} \mathcal{M}_{(\chi, l, 0)}^{\mathrm{BS}, t}=\mathcal{M}_{(\chi, l, 0)}^{\mathrm{GS}}
$$

for all $t>0$. Then $[E] \in \mathcal{M}_{(0, l, \chi)}^{\mathrm{GS}}$ is $\mathrm{IT}_{0}$ if and only if $[\Phi(E)[1]] \in \mathcal{M}_{-(\chi,-l, 0)}^{\mathrm{BS}, t} \cap$ $\mathcal{M}_{(\chi,-l, 0)}^{\mathrm{GS}}[1]$, which holds if and only if $\Delta \Phi(E) \in \mathcal{M}_{(\chi, l, 0)}^{\mathrm{GS}}$ is locally free. But, since all representative sheaves of $\mathcal{M}_{(\chi, l, 0)}^{\mathrm{GS}}$ must be $\mu$-stable, we see that there are nonlocally free sheaves in $\mathcal{M}_{(\chi, l, 0)}^{\mathrm{GS}}$ if and only if $\mathcal{M}_{(\chi, l, 1)}^{\mathrm{GS}} \neq \emptyset$. This happens exactly when the Bogomolov inequality holds for the Chern character $(\chi, l, 1)$, in other words, when $\chi \leq d$, as required.

\section{Polarization line bundles}

Let $L$ be a polarization line bundle on an abelian surface $S$ such that $\operatorname{NS}(S)=\langle L\rangle$. Let $c_{1}(L)=l$, and let $l^{2}=2 d$. In this section we will prove some lemmas that help us to find the value of $\phi_{L}(n)$. We start with the case $n=1$.

PROPOSITION 4.1

If $L$ is an ample line bundle with $c_{1}(L)^{2}=2 d$ on an abelian surface $X$, then

$$
\phi_{L}(1)=\left\lfloor\frac{d-3}{2}\right\rfloor \text {. }
$$

Proof

The Chern character of $E=L \otimes \mathcal{I}_{X}$ is $(1, l, d-|X|)$. Then $\operatorname{ch}(\Phi(E))=(d-|X|$, $-l, 1)$. Such objects are all locally free sheaves exactly when there are no stable sheaves with Chern character $(d-|X|,-l, 2)$. These exist exactly when the Bogomolov inequality holds for such a Chern character (see Definition 3.1). This gives us the criterion $2(d-|X|) \leq d$, so $|X| \geq d / 2$. Hence, $\mathcal{M}_{(1, l, d-|X|)}^{\mathrm{GS}}$ is $\mathrm{IT}_{0}$ if and only if $|X| \leq\lfloor(d-1) / 2\rfloor$. Then $\phi_{L}(1)=\lfloor(d-3) / 2\rfloor$.

The $n=1$ case is exceptional, and we now assume that $n>1$ and find an upper bound for $\phi_{L}(n)$.

\section{PROPOSITION 4.2}

Let $(S, L)$ be a polarized abelian surface such that $\mathrm{NS}(S)=\langle L\rangle$ and $c_{1}(L)^{2}=2 d$. Then $\phi_{L}(n) \leq 2(n-1) d-2$ for $n>1$.

\section{Proof}

By Lemma 3.5, there is $Q$ with Chern character $\operatorname{ch}(Q)=(0, l, d)$ which is not $\mathrm{IT}_{0}$. Since $\chi\left(L^{-n+1} \otimes Q\right)=d(3-2 n)<0$ for $n>1$, we have $\operatorname{Ext}^{1}\left(Q, L^{n-1}\right) \neq 0$. Pick a nontrivial extension

$$
0 \rightarrow L^{n-1} \rightarrow E \rightarrow Q \rightarrow 0
$$


and suppose that $T \hookrightarrow E$ is its torsion subsheaf. Then we have the following diagram:

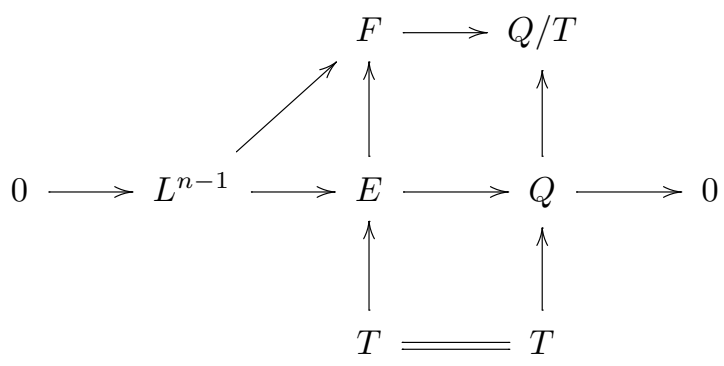

Then $Q / T$ must be supported in dimension 0. But then $\operatorname{Ext}^{1}\left(Q / T, L^{n-1}\right)=0$ and so $L^{n-1} \rightarrow F \rightarrow Q / T$ must split, which is impossible as $F$ is torsion-free and $Q / T$ is torsion. Hence, $T=0$. Then $E \cong L^{n} \otimes \mathcal{I}_{X}$ for some $X$ of length $|X|=2 d(n-1)$ and $E$ is not $\mathrm{IT}_{0}$.

The following theorem proves that the upper bound of $\phi_{L}(n)$ in Proposition 4.2 is sharp.

\section{THEOREM 4.3}

Let $(S, L)$ be a polarized abelian surface with $\mathrm{NS}(S)=\langle L\rangle$ and $c_{1}(L)^{2}=2 d$. Then $\phi\left(L^{n}\right)=2 d(n-1)-2$.

\section{Proof}

By Proposition 4.2 we need to show that $\phi_{L}(n) \geq 2(n-1) d-2$, and we do this by showing that $\mathcal{M}_{\left(1, n l, n^{2} d-k\right)}^{\mathrm{BS}, t}$ is $\mathrm{IT}_{0}$ for all $t$ and $k=2 d(n-1)-1$. Suppose that $E \cong L^{n} \otimes \mathcal{I}_{X}$, where $|X|=2 d(n-1)-1$ is not $\mathrm{IT}_{0}$, and suppose that $\hat{E}:=\Phi(E)$ is $\sigma_{t}$-stable for all $t \gg 0$. Then $\hat{E}$ is a two-step complex such that $H^{-1}(\hat{E})$ is Gieseker stable and $H^{0}(\hat{E})$ is in the form $\mathcal{O}_{Z}$, by Proposition 2.3. The Chern character $\operatorname{ch}\left(H^{-1}(\hat{E})\right)=\left((n-1)^{2} d+d+1,-n, 1+|Z|\right)$. By the Bogomolov inequality

$$
\left((n-1)^{2} d+d+1\right)(1+|Z|) \leq n^{2} d
$$

Therefore

$$
|Z| \leq \frac{2 d(n-1)-1}{d n^{2}-2 d(n-1)+1} .
$$

But $d(n-2)^{2}+2>0$ and so

$$
d n^{2}-2 d(n-1)+1>2 d(n-1)-1 .
$$

Hence, $|Z|<1$. Therefore $H^{0}(\hat{E})=0$ and so $E$ is $\operatorname{IT}_{0}$. If $E$ is $\sigma_{t}$-stable for all $t$, then it follows that $\Phi(E)$ is $\sigma_{t}$-stable for all $t$ (and so also for $t \gg 0$ ). This happens when there are no walls. Unfortunately, there are walls in general. To finish the proof we will identify all the walls and show that all $\sigma_{t}$-semistable objects are $\mathrm{IT}_{0}$ directly. 


\section{LEMMA 4.4}

If $e \in \mathcal{A}_{0}$ destabilizes $L^{n} \otimes \mathcal{I}_{X}$ with $|X|=2 d(n-1)-1$, then $e$ is a rank 1 torsion-free sheaf.

Proof

By Remark 2.2, $H^{-1}(e)=0$ and $E:=H^{0}(e) \cong e$ is torsion-free. Suppose that $\operatorname{ch}(E)=\left(r, g^{\prime} l, \chi\right)$, and let $q=L^{n} \otimes \mathcal{I}_{X} / E$ in $\mathcal{A}_{0}$. Then we have a long exact sequence in $\operatorname{Coh}(S)$ :

$$
0 \rightarrow H^{-1}(q) \rightarrow E \rightarrow L^{n} \otimes \mathcal{I}_{X} \rightarrow H^{0}(q) \rightarrow 0 .
$$

Since $H^{-1}(q) \in \mathcal{F}_{0}$ and $E \in \mathcal{A}_{0}$, we have $\mu(E) \geq 0 \geq \mu\left(H^{-1}(Q)\right)$. Then there is an integer $g>0$ such that $c_{1}(E)=(n r-g) \ell$. Then $c_{1}\left(H^{-1}(Q)\right)=(n r-g-n+m) \ell \leq$ 0 , where $m \geq 0$. Therefore $0<n r-g \leq n-m \leq n$. Hence, $c_{1}(E)$ can be written as $c_{1}(E)=(n-c) \ell$ for some positive integer $c<n$. Since we can assume that $E$ is Bridgeland stable, it must be simple and so the Bogomolov inequality holds (as this is just the statement that the moduli space of simple torsion-free sheaves has dimension at least 2). Consequently, we can write

$$
\chi(E)=\frac{(n-c)^{2} d}{r}-k,
$$

for some rational number $k \geq 0$. Since $E$ is a destabilizer of $L^{n} \otimes \mathcal{I}_{X}$, we have $f\left(E, L^{n} \otimes \mathcal{I}_{X}\right)>0$. Therefore, from condition (2.5), we get

$$
\left((n-c)^{2} d-k r\right) n-\left(n^{2} d-2 d n+2 d+1\right)(n-c) r>0 .
$$

Rearranging (4.3), we obtain

$$
(n-c)\left(-(n-1)^{2} d r-d r-r+d n^{2}-c d n\right)>k r n>0 .
$$

As $n-c>0$, we get walls if $-(n-1)^{2} d r-d r-r+d n^{2}-c d n>0$, so

$$
\frac{1}{r}>\left(1-\frac{1}{n}\right)^{2}+\frac{1}{n^{2}}+\frac{1}{d n^{2}} \geq \frac{1}{2},
$$

for all $n$, since $d>0$. Hence, $r=1$.

\section{REMARK 4.5}

The previous lemma proved that the Chern character of any destabilizer of $L^{n} \otimes$ $\mathcal{I}_{X}$ is given by $\operatorname{ch}(E)=\left(1, n-c,(n-c)^{2} d-k\right)$, which means that $E$ is in the form $L^{n-c} \otimes \mathcal{P}_{\hat{x}} \otimes \mathcal{I}_{Y}$, for some $\hat{x} \in \hat{S}$ and $|Y|=k$.

\section{LEMMA 4.6}

If $L^{n-m} \otimes \mathcal{P}_{\hat{x}} \otimes \mathcal{I}_{Y}$ destabilizes $L^{n} \otimes \mathcal{I}_{X}$, where $|X|=2 d(n-1)-1$, then $m=1$.

Proof

We assume, without loss of generality, that $\hat{x}=0$. Suppose that $F=L^{n-m} \otimes \mathcal{I}_{Y}$ with $|Y|=k$ destabilizes $E=L^{n} \otimes \mathcal{I}_{X}$. Then $\mu(F)-\mu(E) \geq 0$. But if $\mu(E)=$ 
$\mu(F)$, then $\mu_{0, t}(E / F)=\infty$ and so $F$ does not destabilize. Therefore, from condition (4.4), we get

$$
(n-m)\left(-(n-1)^{2} d r-d r-r+d n^{2}-d n m\right)>k r n .
$$

Then we get walls if and only if

$$
\left(1-\frac{m}{n}\right)(2 d n-d n m-2 d-1)>k \geq 0 .
$$

Since $1-m / n$ is positive, this happens if and only if $2 d n-d n m-2 d>1$ and then $2 \geq 2-(d+1) /(n d)>m>0$. Hence, $m=1$.

\section{LEMMA 4.7}

If $L^{n-1} \otimes \mathcal{P}_{\hat{x}} \otimes \mathcal{I}_{Y}$ destabilizes $L^{n} \otimes \mathcal{I}_{X}$ for some $X$ where $|X|=2 d(n-1)-1$, then $|Y|<d(n-2)-1 \leq 2 d(n-2)-1$.

\section{Proof}

Without loss of generality we assume $\hat{x}=0$. Take $F, E$ as in Lemma 4.6. Then from (4.5) we get

$$
\left(1-\frac{1}{n}\right)(d n-2 d-1)>|Y| \geq 0 .
$$

Since $0<1-1 / n<1$, we have $d n-2 d-1>|Y|$.

To complete the proof of Theorem 4.3, we now induct on $n \geq 2$. If $n=2$, then $d(n-2)=0$ and so there are no walls, which establishes the result for the case $n=2$.

Suppose that the statement is true for $n-1 \geq 2$, that is, $L^{n-1} \otimes \mathcal{I}_{X}$ is $\mathrm{IT}_{0}$ for all $X$ with $|X|=2 d(n-2)-1$. To prove that $L^{n} \otimes \mathcal{I}_{X}$ is $\mathrm{IT}_{0}$ for all $X$ with $|X|=2 d(n-1)-1$, we know that the only possible walls are given by $L^{n-1} \otimes \mathcal{I}_{Y}$, where $|Y|<2 d((n-1)-1)-1$. Then there is a short exact sequence

$$
0 \rightarrow L^{n-1} \otimes \mathcal{I}_{Y} \rightarrow L^{n} \otimes \mathcal{I}_{X} \rightarrow Q \rightarrow 0 .
$$

By induction, $L^{n-1} \otimes \mathcal{I}_{Y}$ is $\mathrm{IT}_{0}$, and by Lemma 3.5, $Q$ is $\mathrm{IT}_{0}$ as well, since

$$
\chi(Q)=-(n-1)^{2} d+|Y|+n^{2} d-2 d(n-1)+1=1+d+|Y| \geq d+1 .
$$

Hence, $L^{n} \otimes \mathcal{I}_{X}$ is $\mathrm{IT}_{0}$ for all $X$ with $|X|=2 d(n-1)-1$.

Acknowledgments. The authors are grateful to Kota Yoshioka for pointing out the work of Hiroyuki Terakawa and to the referees for a number of useful comments and corrections.

\section{References}

[AB] D. Arcara and A. Bertram, "Reider's theorem and Thaddeus pairs revisited" in Grassmannians, Moduli Spaces and Vector Bundles, Clay Math. Proc. 14, Amer. Math. Soc., Providence, 2011, 51-68. MR 2807848. 
[BS] T. Bauer and T. Szemberg, Primitive higher order embeddings of abelian surfaces, Trans. Amer. Math. Soc. 349, no. 4 (1997), 1675-1683. MR 1376538. DOI 10.1090/S0002-9947-97-01737-6.

[BM] A. Bayer and E. Macrì, Projectivity and birational geometry of Bridgeland moduli spaces, J. Amer. Math. Soc. 27 (2014), 707-752. MR 3194493. DOI 10.1090/S0894-0347-2014-00790-6.

[BFS] M. Beltrametti, P. Francia, and A. J. Sommese, On Reider's method and higher order embeddings, Duke Math. J. 58 (1989), 425-439. MR 1016428. DOI 10.1215/S0012-7094-89-05819-5.

[BS1] M. Beltrametti and A. J. Sommese, "Zero cycles and $k$ th order embeddings of smooth projective surfaces" with an appendix by L. Göttsche in Problems in the Theory of Surfaces and their Classification (Cortona, 1988), Sympos. Math. 32, Academic Press, London, 1991, 33-48. MR 1273371.

[BS2] _ "On $k$-jet ampleness" in Complex Analysis and Geometry, Univ. Ser. Math., Plenum, New York, 1993, 355-376. MR 1211891.

[Bri] T. Bridgeland, Stability conditions on K3 surfaces, Duke Math. J. 141 (2008), 241-291. MR 2376815. DOI 10.1215/S0012-7094-08-14122-5.

[Huy1] D. Huybrechts, Fourier-Mukai Transforms in Algebraic Geometry, Oxf. Math. Monogr., Oxford Univ. Press, Oxford, 2006. MR 2244106. DOI 10.1093/acprof:oso/9780199296866.001.0001.

[Huy2] Derived and abelian equivalence of K3 surfaces, J. Algebraic Geom. 17 (2008), 375-400. MR 2369091. DOI 10.1090/S1056-3911-07-00481-X.

[HL] D. Huybrechts and M. Lehn, The Geometry of Moduli Spaces of Sheaves, 2nd ed., Cambridge Math. Libr., Cambridge Univ. Press, Cambridge, 2010. MR 2665168. DOI 10.1017/CBO9780511711985.

[Mac] A. Maciocia, Computing the walls associated to Bridgeland stability conditions on projective surfaces, Asian J. Math. 18 (2014), 263-279. MR 3217637. DOI 10.4310/AJM.2014.v18.n2.a5.

[MM] A. Maciocia and C. Meachan, Rank 1 Bridgeland stable moduli spaces on a principally polarized abelian surface, Int. Math. Res. Not. IMRN 2013, no. 9, 2054-2077. MR 3053413.

[MYY] H. Minamide, S. Yanagida, and K. Yoshioka, Some moduli spaces of Bridgeland's stability conditions, Int. Math. Res. Not. IMRN 2014, no. 19, 5264-5327. MR 3267372.

[Muk] S. Mukai, Semi-homogeneous vector bundles on an Abelian variety, Kyoto J. Math. 18 (1978), 239-272. MR 0498572.

[Mum] D. Mumford, Abelian Varieties, Tata Inst. Fund. Res. Stud. Math. 5, Oxford Univ. Press, London, 1974. MR 0282985.

[PP1] G. Pareschi and M. Popa, Regularity on abelian varieties, I, J. Amer. Math. Soc. 16 (2003), 285-302. MR 1949161. DOI 10.1090/S0894-0347-02-00414-9.

[PP2] - Regularity on abelian varieties, II: Basic results on linear series and defining equations, J. Algebraic Geom. 13 (2004), 167-193.

MR 2008719. DOI 10.1090/S1056-3911-03-00345-X. 
[T1] H. Terakawa, Higher order embeddings of algebraic surfaces of Kodaira dimension zero, Math. Z. 229 (1998), 417-433. MR 1658577. DOI $10.1007 /$ PL00004664.

[T2] - The $k$-very ampleness and $k$-spannedness on polarized abelian surfaces, Math. Nachr. 195 (1998), 237-250. MR 1654705. DOI 10.1002/mana.19981950113.

[YY] S. Yanagida and K. Yoshioka, Bridgeland's stabilities on abelian surfaces, Math. Z. 276 (2014), 571-610. MR 3150219.

DOI 10.1007/s00209-013-1214-1.

[Y] K. Yoshioka, Moduli spaces of stable sheaves on abelian surfaces, Math. Ann. 321 (2001), 817-884. MR 1872531. DOI 10.1007/s002080100255.

Alagal: School of Mathematics, University of Edinburgh, Edinburgh, Scotland, United Kingdom; W.Alagal@sms.ed.ac.uk

Maciocia: School of Mathematics, University of Edinburgh, Edinburgh, Scotland, United Kingdom; A.Maciocia@eda.c.uk 